\title{
Chemical Transformations of the Vinyl Group in Natural Chlorins. The Synthesis of 3-Azidomethylchlorins
}

\author{
Marya E. Nikolaeva, ${ }^{a}$ Alexander A. Popov, ${ }^{a}$ Andrei V. Nechaev, ${ }^{\mathrm{a}, \mathrm{b}}$ \\ and Andrey F. Mironov ${ }^{\mathrm{a}}$ \\ ${ }^{a}$ Lomonosov Institute of Fine Chemical Technologies, MIREA - Russian Technological University, 119571 Moscow, Russia \\ ${ }^{\mathrm{b}}$ Federal Scientific Research Centre "Crystallography and Photonics”' of Russian Academy of Sciences, 119333 Moscow, \\ Russia \\ ${ }^{\circledR}$ Corresponding author E-mail: chemorg@mail.ru
}

Transformation of 3-vinylchlorins into azidomethyl-derivatives was investigated for chlorin $p_{6}$ trimethyl ester. The azide group allows modifying chlorophyll derivatives on pyrrole ring A by the use of "click reaction" with terminal alkynes. As an example, reaction with phenylacetylene was carried out.

Keywords: Natural chlorins, chlorin $p_{6}$, azides, photosensitizers, click reaction.

\section{Химические превращения винильной группы в природных хлоринах. Синтез 3-азидометилхлоринов}

\author{
М. Е. Николаева, ${ }^{a}$ А. А. Попов, ${ }^{a}$ А. В. Нечаев, ${ }^{\text {a,b@ }}$ А. Ф. Миронов ${ }^{\mathrm{a}}$ \\ а Институт тонких химических технологий им. М.В. Ломоносова, МИРЭА - Российский технологический \\ университет, 119571 Москва, Россия \\ ${ }^{\mathrm{b}}$ Федеральный научно-исследовательский изентр «Кристаллография и Фотоника» Российской академии наук, 119333 \\ Москва, Россия \\ @E-mail: chemorg@mail.ru
}

\begin{abstract}
Превращение винильной группы в азидометильную в природных хлоринах было изучено на примере тримети-

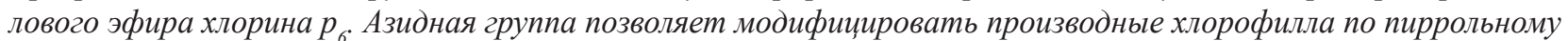
ичклу А с помощью клик-реакичи с терминальными ацетиленами. В качестве примера была проведена реакция с фенилацетиленом.
\end{abstract}

Ключевые слова: Природные хлорины, хлорин $p_{6}$, азиды, фотосенсибилизаторы, клик-реакция.

Photodynamic therapy (PDT) of cancer is widely used in clinical medicine for treatment of malignant tumors. Further successful development of this non-invasive method is closely associated with the development of new highly effective photosensitizers (PS).$^{[1-4]}$ One of the most perspective sources of receiving modern PS is the natural chlorophyll $a$. It can be easily isolated from Spirulina platensis biomass. The possibility of macrocycle substituents modification in chlorophyll derivatives opens the ways for creation of new PS with the improved physical, chemical and spectral properties. ${ }^{[5]}$ One of the directions in the development of drugs on chlorophyll $a$ is its chemical modification, which allows to optimize the hydrophilic-hydrophobic balance of macrocycle substituents, affecting the accumulation of PS in the tumor. ${ }^{[6-7]}$

Chlorin $e_{6}-$ chlorophyll $a$ derivative, the structure of which underlies a number of PSs currently used in oncology (Talaporfin, Photoditazin, etc.). At the same time, chlorin $p_{6}$ and its derivatives, having a similar structure, are not almost used in PDT. Chlorin $p_{6}$ can be easily obtained from purpurin 18 and, unlike chlorin $e_{6}$, contains only one aliphatic carboxyl group on which chemical transformations can be carried out without affecting the two aromatic acid residues.

In order to expand the possible range of PS based on natural chlorins, we have modified the pyrrole ring $\mathrm{A}$ in chlorin $p_{6}$ with the formation of alkylazide group for

(C) ISUCT Publishing 389 


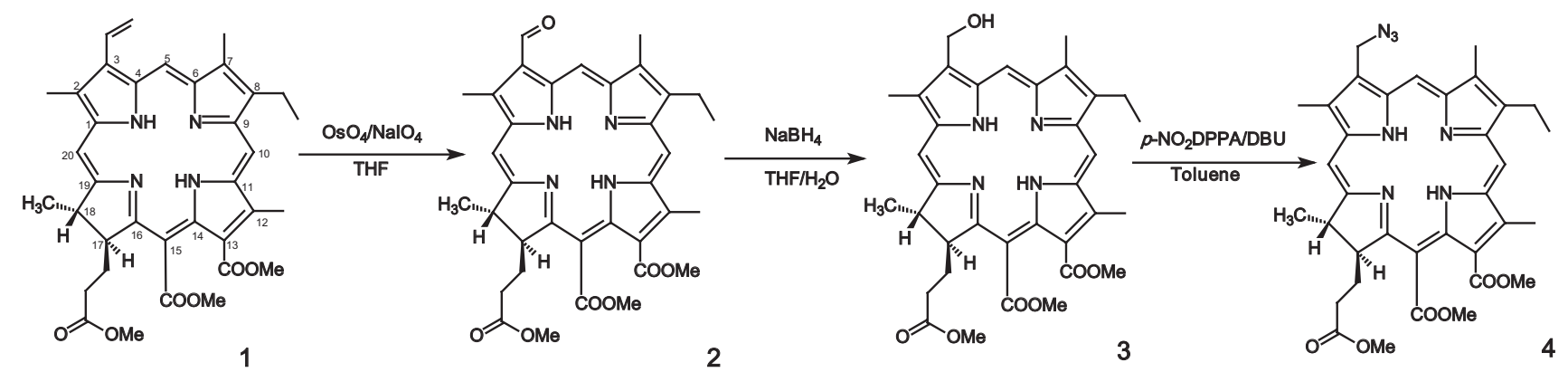

Figure 1. Synthesis of chlorin $p_{6}$ azide derivative.
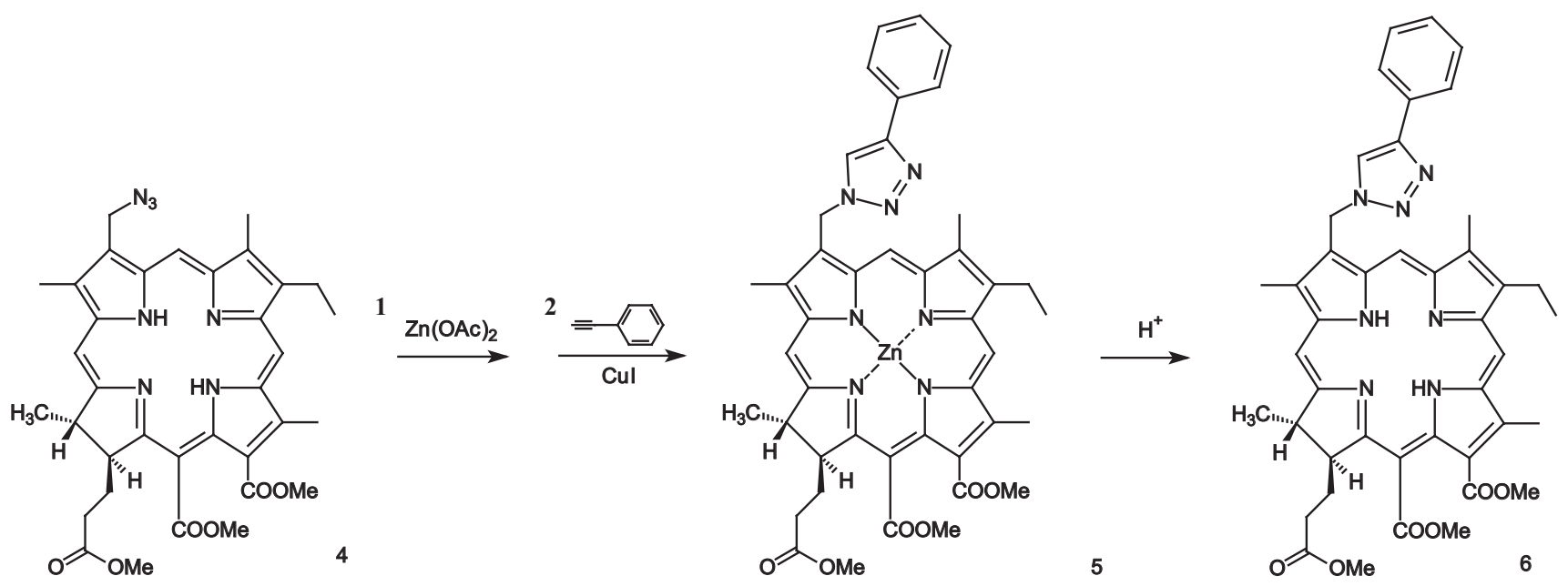

Figure 2. Click reaction.

further modification of molecule by substituents of different nature, affecting the physico-chemical properties of new PS and their tropism to malignant tumors.

The vinyl group in chlorin $p_{6}$ was oxidized to aldehyde (2) by osmium tetroxide in the presence of sodium periodate (Figure 1).

Next, the aldehyde group was reduced to alcohol. In the literature, the complex of tert-butylamine and borane or sodium cyanoborohydride is most frequently used for reduction of 3-formylchlorins with average yields. ${ }^{[8-9]}$ Here, we managed to reduce the aldehyde group in chlorin (2) using solid sodium borohydride in aqueous THF with excellent yield.

Thereafter, a one-step conversion of alcohol to azide was performed. It is known that reaction of bis $(p$-nitrophenyl)phosphorazidate in the presence of DBU with aliphatic alcohols leads to formation of alkylazides. ${ }^{[10]}$ In our case, the alcohol (3) was mixed with excess of $p$ - $\mathrm{NO}_{2} \mathrm{DPPA}$ and DBU in toluene to obtain azide (4). The reaction was fully completed in $10 \mathrm{~min}$ at room temperature with $88 \%$ yield. The structure of the compound was confirmed by ${ }^{1} \mathrm{H}$ NMR spectrum and high-resolution mass spectrum.

Resulting azide (4) easily enters to "click reaction" with compounds containing a terminal acetylene group (Figure 2). Model synthesis was carried out in DMF on a zinc complex of chlorin (4) with phenylacetylene in the presence of copper(I) iodide as a catalyst. The reaction yield was $75 \%$.
Thus, the reported here method of preparation of 3-azidomethylchlorins opens up new possibilities for modification of natural chlorins in pyrrole ring A and the development of new potential photosensitizers for PDT.

\section{Experimental}

Sodium periodate, sodium borohydride and 1,8-diazabicyclo[5.4.0] undec-7-ene (DBU) were purchased from Sigma-Aldrich company. Chlorin $p_{6}$ trimethyl ester, ${ }^{[1]}$ bis( $p$-nitrophenyl)phosphorazidate ${ }^{[12]}$ were prepared according to reported procedures. Column chromatography and preparative TLC was performed on silica gel (Merck, Kieselgel 60, 40-63 $\mu \mathrm{m}$, and 5-40 $\mu \mathrm{m}$, respectively). UV-Visible absorption spectra were taken on a Shimadzu UV-1800 spectrophotometer. ${ }^{1} \mathrm{H}$ NMR spectra were obtained on a $300 \mathrm{MHz}$ Bruker DPX-300 spectrometer. High resolution mass spectra (HRMS) were measured on a Bruker micrOTOF II instrument using electrospray ionization (ESI).

3-Devinyl-3-formylchlorin $p_{6}$ trimethyl ester (2). Chlorin $p_{6}$ trimethyl ester (1) (100 mg, $0.136 \mathrm{mmol})$ was dissolved in $20 \mathrm{ml}$ of THF, $5 \mathrm{ml}$ of $10 \%$ aqueous solution of sodium periodate and $5 \%$ solution of osmium tetroxide in dichloromethane ( $40 \mu 1$, $0.0079 \mathrm{mmol}$ ) was added. The solution was stirred for $1.5 \mathrm{~h}$ at room temperature in argon atmosphere. The mixture was diluted with $100 \mathrm{ml}$ of dichloromethane and washed with water. Organic phase was dried over anhydrous sodium sulfate and evaporated to dryness. The residue was purified by silica gel column chromatography $\left(\mathrm{CHCl}_{3}: \mathrm{MeOH}, 60: 1\right)$. Yield: $84 \mathrm{mg}$ (85\%). 
UV-Vis $\left(\mathrm{CH}_{2} \mathrm{Cl}_{2}\right) \lambda_{\max }\left(\varepsilon / \mathrm{dm}^{3} \mathrm{~mol}^{-1} \mathrm{~cm}^{-1}\right) \mathrm{nm}: 699.0$ (42600), 639.5 (6300), 544.5 (9000), 508.0 (7900), 412.5 (123600). ${ }^{1} \mathrm{H}$ NMR $\left(300 \mathrm{MHz}, \mathrm{CDCl}_{3}\right) \delta_{\mathrm{H}} \mathrm{ppm}: 11.45$ (s, $\left.1 \mathrm{H},-\mathrm{CHO}\right), 10.19$ (s, $1 \mathrm{H}$, $10-\mathrm{H}), 9.68(\mathrm{~s}, 1 \mathrm{H}, 5-\mathrm{H}), 8.84(\mathrm{~s}, 1 \mathrm{H}, 20-\mathrm{H}), 5.19(\mathrm{~d}, J=9.1 \mathrm{~Hz}$, $1 \mathrm{H}, 18-\mathrm{H}), 4.46(\mathrm{~m}, 1 \mathrm{H}, 17-\mathrm{H}), 4.27\left(\mathrm{~s}, 3 \mathrm{H}, 13-\mathrm{CO}_{2} \mathrm{CH}_{3}\right), 4.21(\mathrm{~s}$, $\left.3 \mathrm{H}, 15-\mathrm{CO}_{2} \mathrm{CH}_{3}\right), 3.73\left(\mathrm{~s}, 3 \mathrm{H}, 12-\mathrm{CH}_{3}\right), 3.71(\mathrm{q}, J=7.6 \mathrm{~Hz}, 2 \mathrm{H}$, $\left.-\mathrm{CH}_{2}-\mathrm{CH}_{3}\right), 3.66\left(\mathrm{~s}, 3 \mathrm{H}, 2-\mathrm{CH}_{3}\right), 3.57\left(\mathrm{~s}, 3 \mathrm{H}, 7-\mathrm{CH}_{3}\right), 3.26(\mathrm{~s}, 3 \mathrm{H}$, $\left.17^{3}-\mathrm{CO}_{2} \mathrm{CH}_{3}\right), 2.46\left(\mathrm{~m}, 1 \mathrm{H}, 17^{3}-\mathrm{H}\right), 2.25\left(\mathrm{~m}, 1 \mathrm{H}, 17^{3}-\mathrm{H}\right), 2.14(\mathrm{~m}$, $\left.1 \mathrm{H}, 17^{2}-\mathrm{H}\right), 1.93\left(\mathrm{~m}, 1 \mathrm{H}, 17^{2}-\mathrm{H}\right), 1.88\left(\mathrm{~d}, J=7.2 \mathrm{~Hz}, 2 \mathrm{H}, 18-\mathrm{CH}_{3}\right)$, $1.69\left(\mathrm{t}, J=7.6 \mathrm{~Hz}, 3 \mathrm{H},-\mathrm{CH}_{2}-\mathrm{CH}_{3}\right),-0.86$ and $-1.36(2 \mathrm{bs}, 2 \mathrm{H}, \mathrm{NH})$. 3-Devinyl-3-hydroxymethylchlorin $p_{6}$ trimethyl ester (3). Chlorin (2) $(70 \mathrm{mg}, 0.082 \mathrm{mmol})$ was dissolved in a mixture of $20 \mathrm{ml}$ of THF and $20 \mu \mathrm{l}$ of water. $3 \mathrm{mg}$ of sodium borohydride $(0.026 \mathrm{mmol})$ was added over $2 \mathrm{~h}$. The mixture was diluted with $200 \mathrm{ml}$ of dichloromethane and washed twice with water. Organic phase was dried over anhydrous sodium sulfate and evaporated to dryness. The chlorin (3) was recrystallized from THF:heptane system (1:5). Yield: $63 \mathrm{mg}$ (90 \%). HRMS (ESI) $\mathrm{m} / \mathrm{z}$ 629.2978, calcd for $\mathrm{C}_{35} \mathrm{H}_{40} \mathrm{~N}_{4} \mathrm{O}_{7} \mathrm{M}^{+}, 629.2969$. UV-Vis $\left(\mathrm{CH}_{2} \mathrm{Cl}_{2}\right) \lambda_{\max }\left(\varepsilon / \mathrm{dm}^{3} \mathrm{~mol}^{-1} \mathrm{~cm}^{-1}\right)$ nm: 664 (44600), 609.5 (5600), 526.5 (4700), 496.5 (11800), 397.5 (145800). ${ }^{1} \mathrm{H}$ NMR $\left(300 \mathrm{MHz}, \mathrm{CDCl}_{3}\right) \delta_{\mathrm{H}} \mathrm{ppm}: 9.64(\mathrm{~s}, 1 \mathrm{H}, 10-\mathrm{H})$, 9.34 (s, $1 \mathrm{H}, 5-\mathrm{H}), 8.65(\mathrm{~s}, 1 \mathrm{H}, 20-\mathrm{H}), 5.62\left(\mathrm{~s}, 2 \mathrm{H}, \mathrm{CH}_{2} \mathrm{OH}\right), 5.20$ (d, $J=9 \mathrm{~Hz}, 1 \mathrm{H}, 18-\mathrm{H}), 4.42(\mathrm{~m}, 1 \mathrm{H}, 17-\mathrm{H}), 4.25\left(\mathrm{~s}, 3 \mathrm{H}, 13-\mathrm{CO}_{2} \mathrm{CH}_{3}\right)$, 4.19 (s, $\left.3 \mathrm{H}, 15-\mathrm{CO}_{2} \mathrm{CH}_{3}\right), 3.63$ (q, $\left.J=7.6 \mathrm{~Hz}, 2 \mathrm{H},-\mathrm{CH}_{2}-\mathrm{CH}_{3}\right), 3.62$ (s, 3H, 12- $\left.\mathrm{CH}_{3}\right), 3.54$ (s, 3H, 2- $\left.\mathrm{CH}_{3}\right), 3.29\left(\mathrm{~s}, 3 \mathrm{H}, 7-\mathrm{CH}_{3}\right), 3.14(\mathrm{~s}, 3 \mathrm{H}$, $\left.17^{3}-\mathrm{CO}_{2} \mathrm{CH}_{3}\right), 2.41\left(\mathrm{~m}, 1 \mathrm{H}, 17^{3}-\mathrm{H}\right), 2.24\left(\mathrm{~m}, 1 \mathrm{H}, 17^{3}-\mathrm{H}\right), 2.08(\mathrm{~m}, 1 \mathrm{H}$, $\left.17^{2}-\mathrm{H}\right), 1.90\left(\mathrm{~m}, 1 \mathrm{H}, 17^{2}-\mathrm{H}\right), 1.88\left(\mathrm{~d}, J=7.2 \mathrm{~Hz}, 3 \mathrm{H}, 18-\mathrm{CH}_{3}\right), 1.65(\mathrm{t}$, $\left.J=7.6 \mathrm{~Hz}, 3 \mathrm{H},-\mathrm{CH}_{2}-\mathrm{CH}_{3}\right),-1.14$ (bs, $\left.2 \mathrm{H}, \mathrm{NH}\right)$.

3-Devinyl-3-azidomethylchlorin $p_{6}$ trimethyl ester (4). Hydroxychlorin (3) $(50 \mathrm{mg}, 0.049 \mathrm{mmol})$ was dissolved in $5 \mathrm{ml}$ of toluene, $32 \mathrm{mg}(0.1 \mathrm{mmol})$ of bis ( $p$-nitrophenyl)phosphorazidate and $16 \mu \mathrm{l}(0.1 \mathrm{mmol})$ of DBU were added. Mixture was stirred at a room temperature for $10 \mathrm{~min}$ in argon atmosphere. Solvent was evaporated, product was dissolved in $50 \mathrm{ml}$ of dichloromethane, washed with water followed by $30 \mathrm{ml}$ of $0.5 \% \mathrm{HCl}$ aqueous solution and water. Then solution was dried over anhydrous sodium sulfate and evaporated to dryness. Chlorin (4) was purified by silica gel column chromatography $\left(\mathrm{CHCl}_{3}: \mathrm{MeOH}, 40: 1\right)$. Yield $45 \mathrm{mg}$ (88\%). HRMS (ESI) $\mathrm{m} / z$ 654.3031, calcd for $\mathrm{C}_{35} \mathrm{H}_{39} \mathrm{~N}_{7} \mathrm{O}_{6}$ $\mathrm{M}^{+}$, 654.3034. UV-Vis $\left(\mathrm{CH}_{2} \mathrm{Cl}_{2}\right) \lambda_{\text {max }}\left(\varepsilon / \mathrm{dm}^{3} \mathrm{~mol}^{-1} \mathrm{~cm}^{-1}\right) \mathrm{nm}: 667.0$ (36500), 611.5 (3300), 527 (4500), 497.0 (6900), 398.0 (127400). ${ }^{1} \mathrm{H}$ NMR $\left(300 \mathrm{MHz}, \mathrm{CDCl}_{3}\right) \delta_{\mathrm{H}} \mathrm{ppm}: 9.73(\mathrm{~s}, 1 \mathrm{H}, 10-\mathrm{H}), 9.40(\mathrm{~s}, 1 \mathrm{H}$, 5-H), $8.72(\mathrm{~s}, 1 \mathrm{H}, 20-\mathrm{H}), 5.51\left(\mathrm{~s}, 2 \mathrm{H}, \mathrm{CH}_{2} \mathrm{~N}_{3}\right), 5.19(\mathrm{~d}, \mathrm{~J}=9 \mathrm{~Hz}, 1 \mathrm{H})$, $4.42(\mathrm{~m}, 1 \mathrm{H}), 4.24\left(\mathrm{~s}, 3 \mathrm{H}, 13-\mathrm{CO}_{2} \mathrm{CH}_{3}\right), 4.19\left(\mathrm{~s}, 3 \mathrm{H}, 15-\mathrm{CO}_{2} \mathrm{CH}_{3}\right)$, 3.73 (q, $\left.J=7.6 \mathrm{~Hz}, 2 \mathrm{H},-\mathrm{CH}_{2}-\mathrm{CH}_{3}\right), 3.66\left(\mathrm{~s}, 3 \mathrm{H}, 12-\mathrm{CH}_{3}\right), 3.54$ (s, $\left.3 \mathrm{H}, 2-\mathrm{CH}_{3}\right), 3.43\left(\mathrm{~s}, 3 \mathrm{H}, 7-\mathrm{CH}_{3}\right), 3.26\left(\mathrm{~s}, 3 \mathrm{H}, 17^{3}-\mathrm{CO}_{2} \mathrm{CH}_{3}\right), 2.41(\mathrm{~m}$, $\left.1 \mathrm{H}, 17^{3}-\mathrm{H}\right), 2.23\left(\mathrm{~m}, 1 \mathrm{H}, 17^{3}-\mathrm{H}\right), 2.09\left(\mathrm{~m}, 1 \mathrm{H}, 17^{2}-\mathrm{H}\right), 1.88(\mathrm{~m}, 1 \mathrm{H}$, $\left.17^{2}-\mathrm{H}\right), 1.88\left(\mathrm{~d}, J=7.2 \mathrm{~Hz}, 3 \mathrm{H}, 18-\mathrm{CH}_{3}\right), 1.70(\mathrm{t}, J=7.6 \mathrm{~Hz}, 3 \mathrm{H}$, $\left.-\mathrm{CH}_{2}-\mathrm{CH}_{3}\right),-0.63$ and -1.21 (bs, $\left.2 \mathrm{H}, \mathrm{NH}\right)$.

3-Devinyl-3-(4-phenyl-1,2,3-triazole-1-yl)methyl chlorin $p_{6}$ trimethyl ester (6). Azide (4) $(35 \mathrm{mg}, 0.055 \mathrm{mmol})$ was dissolved in $5 \mathrm{ml}$ of chloroform and $1 \mathrm{ml}$ of methanol. Zinc acetate $(10 \mathrm{mg})$ was added and mixture was stirred for $2 \mathrm{~h}$. Solution was washed with water, dried over anhydrous sodium sulfate and evaporated to dryness. Residue was dissolved in $3 \mathrm{ml}$ of DMF, $10 \mathrm{mg}$ $(0.1 \mathrm{mmol})$ of phenylacetylene and $0.2 \mathrm{mg}$ of copper iodide (I) were added. Mixture was stirred for $2 \mathrm{~h}$ at room temperature. The solvent was evaporated in vacuo, the residue was dissolved in $3 \mathrm{ml}$ of chloroform and $500 \mu \mathrm{l}$ of trifluoroacetic acid was added. Mixture was stirred during $2 \mathrm{~h}, 50 \mathrm{ml}$ of chloroform was added and washed with water, followed by $2 \%$ aqueous solution of $\mathrm{NaHCO}_{3}$ and water. Chlorin (5) was purified by silica gel preparative TLC chromatography $\left(\mathrm{CHCl}_{3}: \mathrm{MeOH}, 40: 1\right)$. Yield $31 \mathrm{mg}$ (75\%). UV-Vis $\left(\mathrm{CH}_{2} \mathrm{Cl}_{2}\right) \lambda_{\max }\left(\varepsilon / \mathrm{dm}^{3} \mathrm{~mol}^{-1} \mathrm{~cm}^{-1}\right) \mathrm{nm}: 669.3(37100)$, 609.3 (3700), 524.9 (4900), 496.2 (7300), 403.6 (129100). ${ }^{1} \mathrm{H}$ NMR $\left(300 \mathrm{MHz}, \mathrm{CDCl}_{3}\right) \delta_{\mathrm{H}}$ ppm: $9.77(\mathrm{~s}, 1 \mathrm{H}, 10-\mathrm{H}), 9.47(\mathrm{~s}, 1 \mathrm{H}, 5-\mathrm{H})$, $8.78(\mathrm{~s}, 1 \mathrm{H}, 20-\mathrm{H}), 7.64(\mathrm{~m}, 2 \mathrm{H}, \mathrm{Ph}-\mathrm{H}), 7.61(\mathrm{~s}, 1 \mathrm{H}$, triazole-H), $7.23(\mathrm{~m}, 3 \mathrm{H}, \mathrm{Ph}-\mathrm{H}), 6.86\left(\mathrm{~s}, 2 \mathrm{H}, 3^{2}-\mathrm{CH}_{2}\right), 5.21(\mathrm{~d}, J=6.2 \mathrm{~Hz}, 1 \mathrm{H}$, $18-\mathrm{H}), 4.45(\mathrm{~m}, 1 \mathrm{H}, 17-\mathrm{H}), 4.26\left(\mathrm{~s}, 3 \mathrm{H}, 13-\mathrm{CO}_{2} \mathrm{CH}_{3}\right), 4.20(\mathrm{~s}, 3 \mathrm{H}$, $\left.15-\mathrm{CO}_{2} \mathrm{CH}_{3}\right), 3.75\left(\mathrm{q}, \mathrm{J}=7.6 \mathrm{~Hz}, 2 \mathrm{H},-\mathrm{CH}_{2}-\mathrm{CH}_{3}\right), 3.68(\mathrm{~s}, 3 \mathrm{H}$, $\left.12-\mathrm{CH}_{3}\right), 3.55\left(\mathrm{~s}, 3 \mathrm{H}, 2-\mathrm{CH}_{3}\right), 3.47\left(\mathrm{~s}, 3 \mathrm{H}, 7-\mathrm{CH}_{3}\right), 3.25\left(\mathrm{~s}, 3 \mathrm{H}, 17^{3}-\right.$ $\left.\mathrm{CO}_{2} \mathrm{CH}_{3}\right), 2.42\left(\mathrm{~m}, 1 \mathrm{H}, 17^{3}-\mathrm{H}\right), 2.24\left(\mathrm{~m}, 1 \mathrm{H}, 17^{3}-\mathrm{H}\right), 2.09(\mathrm{~m}, 1 \mathrm{H}$, $\left.17^{2}-\mathrm{H}\right), 1.90\left(\mathrm{~m}, 1 \mathrm{H}, 17^{2}-\mathrm{H}\right), 1.88\left(\mathrm{~d}, J=7.2 \mathrm{~Hz}, 3 \mathrm{H}, 18-\mathrm{CH}_{3}\right), 1.70$ $\left(\mathrm{t}, J=7.6 \mathrm{~Hz}, 3 \mathrm{H},-\mathrm{CH}_{2}-\mathrm{CH}_{3}\right),-1.3$ (bs, $\left.2 \mathrm{H}, \mathrm{NH}\right)$.

Acknowledgements. This work was supported by the RSF grant No.18-03-00961. High resolution mass spectra were recorded in the Department of Structural Studies of Zelinsky Institute of Organic Chemistry, Moscow.

\section{References}

1. Bonnett R. CRC Press, Boca Raton, FL 2000.

2. Celli J.P., Spring B.Q., Rizvi I., Evans C.L., Samkoe K.S., Verma S., Pogne B.W., Hasan T. Chem. Rev. 2010, 110, $2795-2838$.

3. Oniszczuk A., Wojtunik-Kulesza K.A., Oniszczuk T., Kasprzak K. Biomed. Pharmacather 2016, 83, 912.

4. Moret F., Reddi E. J. Porphyrins Phthalocyanines 2017, 21, 239-256.

5. Mironov A.F. Ross. Khim. Zh. 2017, 61, 42-68 (in Russ.).

6. Jinadasa R.G.W., Hu X., Vicente M.G.H., Smith K.M. J. Med. Chem. 2011, 54, 7464-7466.

7. Suvorov N.V., Machulkin A.E., Ivanova A.V., Popkov A.M., Bondareva E.A., Plotnikova E.A., Yakubovskaya R.I., Majouga A.G., Mironov A.F., Grin M.A. J. Porphyrins Phthalocyanines 2018, 22, 1030-1038.

8. Osuka A., Wada Y., Shinoda S. Tetrahedron 1996, 52, 4311-4326.

9. Sasaki S., Tamiaki H. J. Org. Chem. 2006, 71, 2648-2654.

10. Mizuno M., Shioiri T. Chem. Commun. 1997, 2165-2166.

11. Brandis A.S., Kozyrev A.N., Mironov A.F. Tetrahedron 1992, 48, 6491-6499.

12. Shioiri T., Yamada S. Chem. Pharm. Bull. 1974, 22, 855-858.

Received 15.07.2019 Accepted 27.11.2019 